\title{
Conservación refrigerada de semillas de Araucaria angustifolia (Bert.) O. Kuntze
}

\author{
Refrigerated storage of Araucaria angustifolia (Bert.) O. Kuntze seeds
}

\author{
A. Chaves. ${ }^{1}$, A. Mugridge ${ }^{1}$, H. FAssola ${ }^{1}$, D. AlegranzA ${ }^{2}$, R. FERnAndeZ ${ }^{2}$ \\ ${ }^{1}$ CIDCA: Centro de Investigación y Desarrollo en Criotecnología de Alimentos. \\ CONICET-Facultad de Ciencias Exactas UNLP. 47 y 116 (CP 1900) La Plata, Prov. Buenos Aires, Argentina. \\ E-mail: mcanon@isis.unlp.edu.ar \\ ${ }^{2}$ INTA: Instituto Nacional de Tecnología Agropecuaria, EEA Montecarlo, CC N 4 (CP3384) Montecarlo, \\ Prov. Misiones, Argentina. E-mail: formonte@inta.gov.ar
}

\section{SUMMARY}

The aim of this work was to determine the appropriate refrigerated storage and modified atmosphere conditions to preserve the viability of Araucaria angustifolia seeds.

Plastic film with different gas permeability (PVC, EVA and D), was used to package the seeds. Each package, was stored at different temperatures $\left(0,4\right.$ and $\left.10^{\circ} \mathrm{C}\right)$. Samples were taken every two months, for germination tests at $27^{\circ} \mathrm{C}$.

The highest germination values were obtained with EVA film; after 210 to 270 storage days while seeds wrapped with PVC lost their viability after 60 storage days. In all conditions tested, germination was totally lost after 330 days of storage.

Key words: Araucaria angustifolia, modified atmosphere, germination.

\section{RESUMEN}

El objetivo de este trabajo fue establecer las condiciones de almacenamiento refrigerado y de atmósfera modificada que se adecuaran para conservar el poder germinativo de las semillas de Araucaria angustifolia (Bert.) O. Kuntze durante un período superior a seis meses. Se realizaron paquetes utilizando películas plásticas con diferentes permeabilidades a los gases (PVC, EVA y D) que se almacenaron a temperaturas de 0,4 y $10^{\circ} \mathrm{C}$. Se realizaron pruebas de poder germinativo, a $27^{\circ} \mathrm{C}$, sobre muestras de semillas extraídas bimestralmente del almacenamiento refrigerado.

Los mayores valores de conservación del poder germinativo (hasta 210-270 días) se obtuvieron cuando se empleó la película EVA, mientras que con la película PVC disminuyó a partir de los 60 días de almacenamiento. En todas las condiciones ensayadas, a los 330 días de almacenamiento refrigerado, el poder germinativo se perdió totalmente.

Palabras claves: Araucaria angustifolia, atmósfera modificada, germinación.

\section{INTRODUCCION}

El contar con técnicas de conservación de semillas de Araucaria angustifolia es una alternativa para sortear el inconveniente causado por la ciclicidad de producción, que provoca años de escasez absoluta de semillas en rodales de la provincia de Misiones, Argentina.
El empleo de técnicas adecuadas de producción de plantines (Fassola y Allegranza 1996), complementadas con técnicas de conservación de semillas permitirá un uso racional de los escasos recursos genéticos locales, que son los que mejor crecimiento exhiben (Fahler 1981).

De acuerdo con Tompsett (1984), cuatro especies del género Araucaria (A. araucana, A. 
angustifolia, A. hunsteinii y A. bidwilii) no pueden ser conservadas con contenidos de humedad por debajo del 25-40\%, por lo que deben ser clasificadas como "recalcitrantes". Específicamente en el caso de Araucaria angustifolia el nivel crítico de humedad, por debajo del cual hay pérdida total de viabilidad, es aproximadamente el $38 \%$ (Eira et al. 1994). Esta característica imposibilita la conservación por largo tiempo de las semillas de esta especie, dado que después de su maduración se inicia el proceso natural de deshidratación.

Hasta el momento, sólo han sido exitosas algunas experiencias de conservación a corto plazo (24 meses), con semillas almacenadas en sacos plásticos, que mantenían su grado de humedad original y una temperatura de 4-6 ${ }^{\circ} \mathrm{C}$ (Prange 1964, Suiter Filho 1966, Ferreira 1977, Shimizu y Oliveira 1983, op. cit. por Eira et al. 1994).

El uso de bolsas plásticas flexibles para el envasado de vegetales evita la deshidratación y permite que en su interior se genere una atmósfera modificada que puede favorecer la conservación del producto. La composición de dicha atmósfera será función de la permeabilidad a los gases de la película empleada, del área disponible para el intercambio gaseoso, de la cantidad de producto envasado y de la actividad respiratoria del mismo (Day 1990). Además, la aplicación de bajas temperaturas permite disminuir la velocidad de los procesos metabólicos del vegetal durante el tiempo de almacenamiento.

Considerando estos antecedentes, el objetivo de este trabajo ha sido estudiar la influencia de diferentes atmósferas modificadas (obtenidas mediante el empleo de películas plásticas con distintos grados de permeabilidad a los gases) y temperaturas de almacenamiento refrigerado sobre el mantenimiento del poder germinativo de semillas de Araucaria angustifolia durante un período superior a seis meses.

\section{MATERIAL Y METODOS}

Material vegetal. Se utilizaron semillas de Araucaria angustifolia (Bert.) O. Kuntze, recolectadas en los rodales implantados ubicados en el Campo Anexo Manuel Belgrano (INTA-EEA Montecarlo), localidad San Antonio, provincia de Misiones, Argentina (situado geográficamente a los $26^{\circ} 04^{\prime}$ de Latitud Sur y $53^{\circ} 45^{\prime}$ de Longitud Oeste, a una altitud de $565 \mathrm{msnm}$ ).
El poder germinativo inicial de las semillas fue evaluado en forma inmediata a la recolección en 1a EEA Montecarlo del INTA, arrojando un valor del $50 \%$; luego, fueron transportadas por vía terrestre hasta el laboratorio del CIDCA (CONICET Fac. Cs. Exactas, UNLP), ubicado en La Plata, provincia de Buenos Aires, Argentina.

En el laboratorio las semillas fueron colocadas, en los envases con que se transportaron desde origen, en una cámara a $4^{\circ} \mathrm{C}$ y después de 24 horas fueron acondicionadas para realizar la experiencia. Este proceso de enfriamiento contribuyó a que, posteriormente, cuando las semillas fueron envasadas en forma definitiva, no se produjera condensación de humedad en el interior del envase.

Acondicionamiento. Aproximadamente $850 \mathrm{~g}$ de semillas (entre 120 y 140), muestreadas al azar, fueron colocadas en bandejas de poliestireno y empaquetadas con películas plásticas flexibles. Las películas utilizadas fueron: cloruro de polivinilo (PVC), etil-vinil-acetato (EVA) y una poliolefina multicapa (D), cuyas características técnicas se muestran en el cuadro 1 .

Almacenamiento. Las temperaturas utilizadas para el almacenamiento fueron 0,4 y $10^{\circ} \mathrm{C}$. Los paquetes conteniendo las semillas fueron colocados en las cámaras de forma tal de no disminuir la superficie de transferencia de la película con el medio. Se extrajeron muestras de todas las temperaturas y tipos de embalaje en forma bimestral. En ninguno de los tratamientos se realizó aplicación de productos antifúngicos.

Composición atmosférica. Se determinaron las concentraciones de $\mathrm{O}_{2}$ y $\mathrm{CO}_{2}$ correspondientes a las distintas atmósferas desarrolladas dentro de los envases. Se tomaron muestras diariamente hasta alcanzar el equilibrio y luego cada 2 meses hasta finalizar el almacenamiento. La muestra gaseosa se tomó con una jeringa a través del film, tapándose la perforación inmediatamente con cinta adhesiva. Se utilizó un cromatógrafo gaseoso Shimadzu con columnas de sílica gel y tamiz molecular 5 A, conectadas en serie, y un detector de conductividad térmica. Como gas transportador se utilizó $\mathrm{H}_{2}$. Los resultados se expresaron en $\mathrm{CO}_{2}$ $(\%)$.

Conteo de semillas germinadas. Luego de cumplido cada tiempo de almacenamiento refrigerado, se 


\section{CUADRO 1}

Características técnicas de las películas plásticas flexibles empleadas.

Technical characteristics of plastic films used.

\begin{tabular}{|c|c|c|c|c|}
\hline $\begin{array}{l}\text { Privado } \\
\text { Película }\end{array}$ & $\begin{array}{c}\text { Permeabilidad } \\
\text { al vapor de } \\
\text { agua* }\end{array}$ & $\begin{array}{l}\text { Permeabilidad al } \\
\mathrm{O}_{2}^{* *}\end{array}$ & $\begin{array}{l}\text { Permeabilidad al } \\
\qquad \mathrm{CO}_{2} * *\end{array}$ & $\begin{array}{c}\text { Espesor } \\
\text { (micrones) }\end{array}$ \\
\hline PVC & 40.0 & 14.232 & 48.552 & 15 \\
\hline EVA & 16.0 & 3.732 & 17.012 & 15 \\
\hline D & 23.5 & 9.500 & 31.000 & 15 \\
\hline
\end{tabular}

* $\mathrm{g} /\left(\mathrm{m}^{2} .24\right.$ horas); a $38^{\circ} \mathrm{C}, 100 \% \mathrm{HR}$ (humedad relativa) ** $\mathrm{cm}^{3} /\left(\mathrm{m}^{2} \cdot 24\right.$ horas 1 atmósfera); a $23^{\circ} \mathrm{C}, 0 \% \mathrm{HR}$ PVC: Cloruro de polivinilo. Producido por Casco S.A.

EVA: Etil-vinil-acetato. Provista por Grace S.A.

D: Poliolefina multicapa, irradiada y coextrudada, termosellable e imprimible. Provista por Grace S.A.

procedió al conteo de las semillas germinadas dentro de los envases realizados con las distintas películas; en base al total de semillas contenidas en cada empaquetamiento se determinó el porcentaje correspondiente. Asimismo, se observó el estado sanitario de las mismas.

Determinación del poder germinativo. El poder germinativo inicial se determinó en forma inmediata al arribo de las semillas al laboratorio del CIDCA; se colocó una muestra de 40 semillas (tomadas de a 10) en bandejas con arena húmeda, en una cámara de germinación a $27^{\circ} \mathrm{C}$. Cada 2 meses de almacenamiento refrigerado se extrajeron muestras de cada condición de temperatura y película y sobre esas semillas se determinó el poder germinativo. Para ello, de cada muestra, se utilizaron 20 semillas que fueron colocadas de a 10 (en 2 bandejas) en la cámara de germinación. Los resultados se expresaron en porcentaje de semillas germinadas.

Para determinar el poder germinativo, después de cada período de almacenamiento refrigerado, no se tuvieron en cuenta las semillas que estaban germinadas en las bolsas. Por ello, los datos informados para poder germinativo se refieren a las semillas que no salieron germinadas de las distintas cámaras de almacenamiento refrigerado.

Para evitar el desarrollo de hongos durante la germinación, después de cada período de almacenamiento y antes de la prueba de germinación, las semillas fueron tratadas con Captan (PM) 20 $\mathrm{g} / 101 \mathrm{de}$ agua por inmersión durante $10 \mathrm{~min}$.
Diseño experimental y análisis estadístico. Las experiencias de almacenamiento y determinaciones de poder germinativo se realizaron usando dos repeticiones. Se empleó un diseño factorial donde los factores tenidos en cuenta fueron: temperatura de almacenamiento, tiempo de almacenamiento refrigerado y película plástica utilizada para el empaquetamiento. Para temperatura se usaron 3 niveles $\left(0,4\right.$ y $\left.10^{\circ} \mathrm{C}\right)$, para tiempos 5 niveles $(60$, $120,210,270$ y 330 días) y para película 3 niveles (PVC, EVA y D). Los datos fueron analizados mediante un ANOVA y las medias se compararon con el test LSD (least significant differences) con un grado de significación $\alpha=0.05$.

\section{RESULTADOS}

Variación de la composición atmosférica del interior del envase durante el almacenamiento refrigerado. La composición atmosférica dentro de los envases cambió rápidamente. Los valores de $\mathrm{CO}_{2}$ de equilibrio alcanzados al cabo de 10 días de almacenamiento y que luego se mantuvieron constantes, fueron los indicados en el cuadro 2. El contenido de $\mathrm{CO}_{2}$ (\%) aumentó especialmente cuando se empleó la película EVA (llegó aproximadamente a $1 \%$ ). El contenido de $\mathrm{O}_{2}$ siempre fue el complementario al $21 \%$.

Efecto de la temperatura de almacenamiento en la germinación de semillas envasadas. $A$ la salida de cámara refrigerada, se observó que había semillas 


\section{CUADRO 2}

$\mathrm{CO}_{2}(\%)$ alcanzado en la atmósfera dentro de los envases realizados con las diferentes películas plásticas flexibles y almacenados a 0,4 y $10^{\circ} \mathrm{C}$.

$\mathrm{CO}_{2}(\%)$ in packages with plastic films stored at 0,4 and $10^{\circ} \mathrm{C}$.

\begin{tabular}{|c|c|c|c|}
\hline \multirow{2}{*}{ Película } & \multicolumn{3}{|c|}{ Temperatura ${ }^{\circ} \mathrm{C}$} \\
\cline { 2 - 4 } & 0 & 4 & 10 \\
\hline PVC & 0.16 & 0.00 & 0.16 \\
EVA & 0.56 & 0.97 & 0.87 \\
D & 0.14 & 0.44 & 0.14 \\
\hline
\end{tabular}

que presentaban germinación en distintos grados de evolución.

En la figura 1 se puede observar el porcentaje de semillas germinadas dentro de los envases en función de la temperatura de almacenamiento, tipo de película empleada y tiempo de almacenamiento refrigerado.

Cuando las semillas se almacenaron a $0^{\circ} \mathrm{C}$, las únicas que mostraron germinación durante el almacenamiento refrigerado fueron las empaquetadas con la película D: $2 \%$ a partir de los 210 días, hasta alcanzar el 6.6 y $6.3 \%$ (valores que no difieren entre sí, p>0.05) a los 270 y 330 días de conservación, respectivamente.

El valor promedio de germinación, a la salida de cámara, para las semillas almacenadas a $4^{\circ} \mathrm{C}$, llegó hasta el $12.6 \%$ a los 270 días, en los empaquetamientos realizados con la película D. Cuando se utilizó la película EVA, sólo se alcanzó un $3 \%$ luego de 330 días de conservación. Las semillas acondicionadas con la película PVC no manifestaron germinación en ningún tiempo de conservación a esta temperatura.

En el almacenamiento realizado a $10^{\circ} \mathrm{C}$ se observó la mayor cantidad de semillas germinadas a la salida de cámara. Cuando se utilizó para los empaquetamientos la película D se registró un 5\% de germinación a partir de los 60 días de refrigeración y se alcanzaron valores de hasta $45 \%$ a los 330 días.

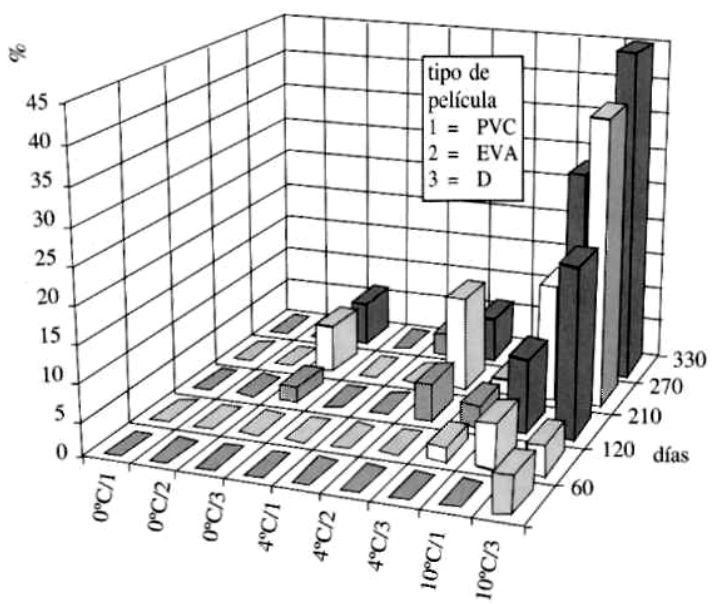

Figura 1. Germinación (\%) dentro de los envases en función de la temperatura, del tipo de película plástica y del tiempo de conservación. LSD: 4.09'

Germination (\%) inside the packages in relation to temperature, plastic film and conservation period.

Las semillas empaquetadas con la película EVA evidenciaron germinación a partir de los 120 días de almacenamiento a $10^{\circ} \mathrm{C}$, llegando a un $28 \%$ a los 330 días. Esta fue la única condición de almacenamiento (película y temperatura) en que, a los 60 días, se pudo observar actividad de orugas de Laspeyressia araucariae (determinada por la presencia de aserrín adherido al extremo de la semilla y disperso en el envase); al prolongarse el período de almacenamiento esta actividad desapareció.

Los valores de germinación registrados, a la salida de cámara, para las semillas refrigeradas a $10^{\circ} \mathrm{C}$ y empaquetadas con PVC fueron del $2 \%$ a partir de los 120 días y se mantuvieron prácticamente constantes hasta el final del almacenamiento (figura 1). Es de resaltar que utilizando para el empaquetamiento este tipo de película, a los 330 días a esta temperatura los brotes se presentaban oscurecidos y secos.

Efecto de las condiciones de almacenamiento en el poder germinativo. El poder germinativo inicial, cuyo valor fue del $50 \%$, fue registrado en el lugar de origen de las semillas y a su arribo al laboratorio del CIDCA.

La evaluación del poder germinativo no incluye las semillas que germinaron dentro de los envases durante el almacenamiento refrigerado. Se consideró que, aunque se subestime el poder germinativo general, no sería conveniente su utilización porque aumenta el descarte (pues la raíz de 
las mismas se puede encontrar deshidratada y además es fácil de romper en el manipuleo previo a la siembra).

En la figura 2 se puede observar la variación de los porcentajes promedios de germinación, obtenidos para las distintas temperaturas, películas y tiempos de almacenamiento refrigerado, utilizados para el ensayo.

Al analizar los datos, se observó que hubo diferencias muy significativas $(\mathrm{p}<0.01)$ en el poder germinativo debido al empleo de las distintas películas de envase, el tiempo de refrigeración y diferentes temperaturas de almacenamiento. En cuanto a las interacciones, las únicas que se manifestaron como muy significativas fueron las que incluyen los tres factores antes mencionados y la de temperatura con película (cuadro 3).

Del resultado del test de $\mathrm{LSD}$, aplicado a distintas películas, surgió que la utilización de la película EVA permitió alcanzar mayor poder germinativo que las otras dos, mientras que no hubo diferencias significativas entre las películas PVC y D (LSD entre películas: 10.26).

Si bien no se encontraron diferencias significativas entre los valores de germinación de las semi- llas almacenadas a 0 y $4^{\circ} \mathrm{C}$, hubo un aumento en el porcentaje cuando se almacenaron a $10^{\circ} \mathrm{C}$, respecto a las otras 2 temperaturas $(\mathrm{LSD}=10.26)$.

El poder germinativo se logró mantener hasta los 270 días de almacenamiento refrigerado, observándose distintos porcentajes de germinación según la temperatura del almacenaje y el tipo de película utilizada para el empaquetamiento. En todas las condiciones de trabajo, luego de 330 días de almacenamiento refrigerado, el poder germinativo se perdió totalmente.

La película EVA permitió mantener, aproximadamente, el valor de germinación inicial (50\%) hasta los 60 días de almacenamiento refrigerado. A partir de los 120 días el comportamiento fue diferente según cual haya sido la temperatura de almacenamiento. El poder germinativo de las semillas almacenadas con esta película, a 0 y $10^{\circ} \mathrm{C}$, aumentó hasta el $75 \%$ a los 120 días, para luego decaer y llegar a valores de 10 y $50 \%$ respectivamente (a los 270 días de almacenamiento refrigerado). A $4^{\circ} \mathrm{C}$ los valores de germinación tuvieron menor variación, alcanzando un $35 \%$ a los 270 días.

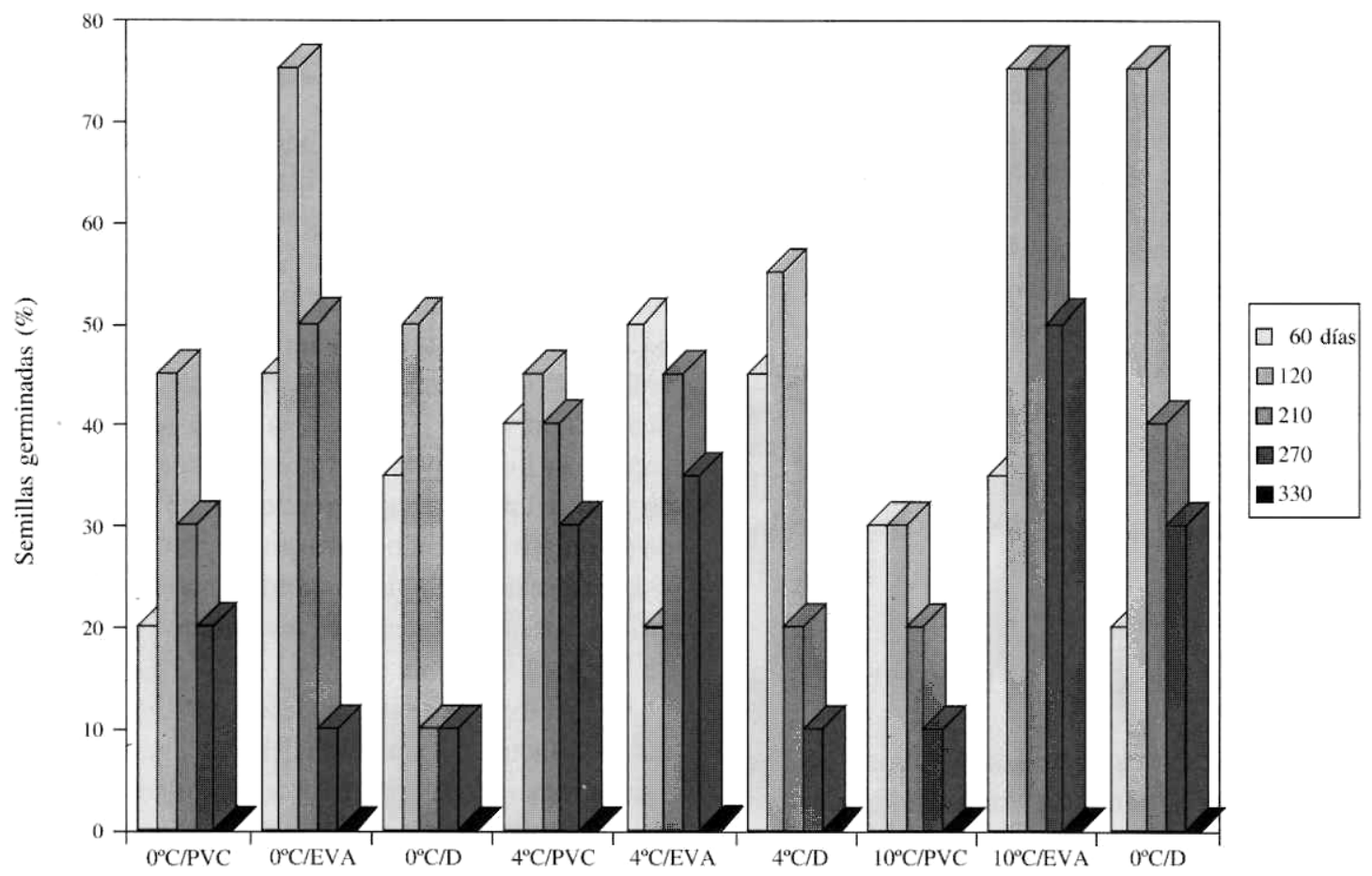

Figura 2. Germinación (\%) a $27^{\circ} \mathrm{C}$ en función del tipo de película plástica, de la temperatura y del tiempo de almacenamiento. LSD: 28.1

Germination (\%) at $27^{\circ} \mathrm{C}$ in relation to plastic film type, temperature and conservation period. 
Análisis de varianza del porcentaje de semillas germinadas a $27^{\circ} \mathrm{C}$ ANOVA of germinated seeds at $27^{\circ} \mathrm{C}$.

\begin{tabular}{|lrrrrr|}
\hline Fuentes de variación & $\begin{array}{c}\text { Grados de } \\
\text { libertad }\end{array}$ & $\begin{array}{r}\text { Suma de } \\
\text { cuadrados }\end{array}$ & $\begin{array}{c}\text { Cuadrado } \\
\text { medio }\end{array}$ & $\begin{array}{c}\text { Valor } \\
\text { de F }\end{array}$ & $\begin{array}{c}\text { Probabi- } \\
\text { lidad }\end{array}$ \\
\hline Repetición & 1 & 7.803 & 7.803 & 0.0947 & \\
Temperatura & 2 & 987.295 & 493.648 & 5.9898 & $0.0050^{*}$ \\
Película & 2 & 1.896 .872 & 948.436 & 11.5082 & $0.0001^{*}$ \\
Temp. x película & 4 & 2.125 .695 & 531.424 & 6.4482 & $0.0004^{*}$ \\
Tiempo & 4 & 19.479 .027 & 4.869 .757 & 59.0889 & $0.0000^{*}$ \\
Temp. x tiempo & 8 & 1.162 .895 & 145.362 & 1.7638 & 0.1105 \\
Película x tiempo & 8 & 883.383 & 110.423 & 1.3399 & 0.2497 \\
Temp.x pelíc.x tiempo & 16 & 4.510 .371 & 281.898 & 3.4205 & $0.0006^{*}$ \\
Error & 44 & 33.626 .218 & 82.414 & & \\
\hline Total & 89 & 34.679 .559 & & & \\
\hline
\end{tabular}

* = Altamente significativo al $99 \%$ de probabilidad.

Cuando se utilizó la película PVC, los valores de germinación inicial se mantuvieron hasta los 120 días cuando las semillas se almacenaron a $0 \mathrm{y}$ $4^{\circ} \mathrm{C}$, decayendo luego hasta el 20 y $30 \%$ respectivamente a los 270 días. A $10^{\circ} \mathrm{C}$ el poder germinativo bajó al $30 \%$ a partir de $\operatorname{los} 60$ días de almacenamiento para continuar descendiendo hasta perderse totalmente.

Cuando los empaquetamientos se realizaron con la película D, las semillas que mantuvieron mejor el poder germinativo fueron las almacenadas a $4 \mathrm{y}$ $10^{\circ} \mathrm{C}$ durante no más de 120 días, momento a partir del cual llegó a valores más bajos a 4 que a $10^{\circ} \mathrm{C}$. La disminución del porcentaje de germinación en las semillas almacenadas a $0^{\circ} \mathrm{C}$ fue notoria, pues ya a los 60 días de conservación el mismo cayó a un $35 \%$.

\section{ANALISIS Y DISCUSION DE RESULTADOS}

La utilización de películas plásticas flexibles y el empleo de bajas temperaturas para el almacenamiento determinaron que se notara un aumento del poder germinativo, respecto al inicial, a partir de los 120 días de almacenamiento hasta los 210 días, especialmente cuando se usaron las películas con menor permeablidad a los gases.
Es de resaltar, por lo tanto, que el mantenimiento de un mayor poder germinativo, cuando las semillas fueron envasadas con la película EVA, estaría ligado a las condiciones que se generaron en el interior del envase.

En relación a la composición de la atmósfera, dentro de los envases realizados con la película EVA, una característica distintiva fue la mayor concentración de $\mathrm{CO}_{2}$ que se produjo en ellos. Aunque el porcentaje de $\mathrm{CO}_{2}$ dentro del envase no explicaría el mayor poder germinativo observado, a $4^{\circ} \mathrm{C}$ se produjo un aumento del $\mathrm{CO}_{2}$ (cuadro 2), tanto para las películas EVA como D (alcanzando los mayores valores de concentración de $\left.\mathrm{CO}_{2}\right)$ y un porcentaje prácticamente nulo cuando se utilizó PVC; sin embargo en promedio, para esa temperatura, la germinación obtenida en este último tipo de envase fue levemente inferior al de EVA.

Este comportamiento podría estar ligado a algún otro factor. La variación del poder germinativo estaría relacionada con la susceptibilidad a la deshidratación u otras características de las semillas. Esto, según Chin (1988) y Berjak y Pammenter (1995), fue encontrado no solamente entre especies, sino también entre poblaciones de una misma especie o semillas de un mismo lote. 
La película PVC, por su parte, con la más alta permeabilidad al vapor de agua, habría permitido una mayor deshidratación de las semillas, lo que podría asociarse a la menor conservación del poder germinativo y escasa germinación dentro del envase.

Tampoco con la temperatura se verificó un comportamiento o una tendencia clara. Según Bonner (1995), a fin de inhibir la germinación durante el almacenamiento de semillas recalcitrantes, debe utilizarse la menor temperatura posible. Sin embargo, en los envases EVA se obtuvo el mínimo de conservación de poder germinativo, promedio de las distintas observaciones, a una temperatura de $4^{\circ} \mathrm{C}$. El mayor porcentaje se logró a $10^{\circ} \mathrm{C}$; a esta temperatura le correspondió un 75 y un $50 \%$ de poder germinativo a $\operatorname{los} 210$ y 270 días respectivamente, aunque también se observaron los valores más altos de semillas germinadas dentro de los envases. Mientras que a $0^{\circ} \mathrm{C}$ para similares períodos de almacenamiento se obtuvo un 50 y un $10 \%$ de conservación del poder germinativo.

Si bien durante el almacenamiento a 10 y $4^{\circ} \mathrm{C}$ el poder germinativo mantuvo valores relativamente altos, la mayor cantidad de semillas germinadas en los envases y la actividad de Laspeyreassia araucariae determinó que estas temperaturas no sean recomendables para la conservación a largo plazo.

Empleando la película EVA, a 0 y $10^{\circ} \mathrm{C}$, como también la película D a 4 y $10^{\circ} \mathrm{C}$, fue posible observar, sobre todo a los 120 días, un poder germinativo superior al inicial $(50 \%)$. De acuerdo con Aquila y Ferreira (1984) en Araucaria angustifolia puede ocurrir una dormancia inicial poco pronunciada, que el almacenamiento por corto plazo, 2 a 4 meses, hace desaparecer. La salida de la dormancia o la pérdida de resistencia a la germinación puede indicar que se está próximo al decrecimiento del vigor de la semilla.

Esto se puede observar en la figura 2, donde se ve que luego de alcanzar los valores máximos de poder germinativo, para las opciones de conservación mencionadas en el párrafo anterior, éste comienza a decrecer para ser totalmente nulo a los 330 días, independientemente del envase y la temperatura de conservación.

\section{CONCLUSIONES}

La respuesta obtenida en el poder germinativo de semillas de Araucaria angustifolia fue diferente cuando fueron conservadas en envases realizados con distintas películas plásticas flexibles, temperaturas y tiempo de almacenaje.

A pesar de la baja permeabilidad al vapor de agua de algunas de las películas empleadas, en ningún caso el período de conservación del poder germinativo fue superior a 10 s 270 días.

La menor permeabilidad al vapor de agua de la película D provocó un mayor porcentaje de semillas germinadas dentro de los envases.

Se desestima el uso de la película PVC, ya que su alta permeabilidad al vapor de agua determinó una importante deshidratación que perjudicó la viabilidad de las semillas.

El porcentaje de semillas germinadas dentro de los envases aumentó cuanto mayor fue la temperatura del almacenamiento.

Transcurridos 330 días de almacenamiento refrigerado, el poder germinativo de las semillas se perdió completamente, para todas las condiciones de temperatura y película de envase ensayadas.

De los resultados obtenidos se puede recomendar, para conservar el poder germinativo de las semillas de Araucaria angustifolia, el uso de la película EVA (u otra con características similares) y una temperatura de almacenaje igual a $0^{\circ} \mathrm{C}$.

\section{BIBLIOGRAFIA}

AQUiLA, A. M.E., A. FERREIRA. (1984). "Germinaçaço de sementes escarificadas de Araucaria angustifolia em solo", Ciencia e Cultura 36 (9): 1583-1587.

BERJAK, P., N.W. PAMMENTER. (1995). Recalcitrant (Desiccation-Sensitive) Seeds. Innovations in Tropical Tree Seed Technology. Proc. IUFRO Symp. Project Group P.2.04.00 "Seed Problems". Arusha, Tanzania: 14-29.

BONNER, F.T. (1995). Storage of temperate recalcitrant forest tree seed. Innovations in Tropical Tree Seed Technology. Proc. IUFRO Symp. Project Group P.2.04.00 "Seed Problems". Arusha, Tanzania.: 30-36.

CHIN, H.F. (1988). Recalcitrant seeds-a status report. Roma, IBPGR, $28 \mathrm{p}$.

DAY, B.P.F. (1990). "A perspective of modified atmosphere packaging of fresh produce in Western Europe", Food Science and Technology Today 4 (4): 215-221.

EIRA, M.T.S., A.N. SAlOMAO, R. DA CUNHA, D.K. CARRARA, C.M.C. MELlO. (1994). "Efeito do teor de agua sobre a germinação de sementes de Araucaria angustifolia (Bert.) O. Kuntze-Araucariaceae", Revista Brasileira de Sementes. 16, (1): 71-75. 
FAHLER, J. (1981). Variación geográfica entre y dentro de orígenes de Araucaria angustifolia a la edad de 8 años en la provincia de Misiones. Tesis de MS Universidade Federal do Paraná. Curitiba. Brasil.

FASSOlA H. E., D. AlegranzA. (1996). Producción de mudas de Araucaria angustifolia Bert. O Kuntze en distin- tos tipos de envase. Informe Técnico $\mathrm{N}^{\circ}$ 9. INTA-EEA Montecarlo.

TOMPSETT, P.B. (1984). "Desiccation studies in relation to the storage of Araucaria seed", Ann. Appl. Biol., 105: 587589 . 